\title{
Tetanus in a post operative patient-A case report
}

\author{
Paul Dienye ${ }^{1}$, Andrew Bock-Oruma ${ }^{1}$, Damien Uyagu ${ }^{2}$ \\ ${ }^{1}$ Department of Family Medicine, University of Port Harcourt Teaching Hospital, Port Harcourt, Nigeria; \\ pdienye@yahoo.com, bock-oruma@yahoo.com \\ ${ }^{2}$ Department of Family Medicine, University of Benin Teaching Hospital, Benin City, Nigeria; damienduyagu@yahoo.com
}

Received 24 January 2013; revised 28 February 2013; accepted 6 March 2013

\begin{abstract}
Tetanus is a preventable disease which commonly occurs with puncture or penetrating wounds, or contamination of cutaneous wounds. It may be secondary to surgical procedures such as gastrointestinal surgery on rare occasions. This article reports a fatal case of tetanus in a post operative patient in which the source of infection could not be ascertained. Active immunization of all persons against tetanus, adequate sterilization of surgical equipment and consumables as well as the operating rooms is advocated.
\end{abstract}

Keywords: Appendectomy; Post Operative Tetanus; Immunisation; Developing Countries

\section{INTRODUCTION}

Tetanus is caused by Clostridium tetani, a spore-forming anaerobic bacterium. This organism produces tetanospasmin which binds to the central nervous system causing diffuse muscle spasms and autonomic instability that characterizes the disease [1]. Tetanus commonly occurs with puncture or penetrating wounds, or contamination of cutaneous wounds. On rare occasions, it may be secondary to surgical procedures such as gastrointestinal surgery. In some occasions, the source of infection may be difficult to determine. This article reports a fatal case of tetanus in a post operative patient.

\section{CASE PRESENTATION}

AK was a 25-year-old seamstress who was referred from a clinic to a rural mission hospital with a diagnosis of tetanus. The referral letter stated that she had presented with a three day history of severe lower abdominal pain with associated fever, nausea and vomiting. Based on their finding of lower abdominal tenderness with guarding at the right iliac fossa, dirty-white vaginal discharge and positive cervical excitation tenderness, diagnoses of acute appendicitis and pelvic inflammatory disease were made. A high vaginal swab was taken for microscopy, culture and sensitivity and she was empirically placed on intravenous ampicillin/cloxacillin, gentamicin and metronidazole. On the third day the lower abdominal pain had localized at the right iliac fossa with rebound tenderness. She had appendectomy done and the diagnosis was confirmed by histopathological examination of the specimen.

Her referral was based on a presumptive diagnosis of tetanus due to the complaint on the first post operative day of her inability to open her mouth while trying to brush her teeth. There was no history of exposure to strychnine-containing substances such as pesticides.

On examination, she was alert and well oriented. Her pulse rate was 78/min and the blood pressure was 120/80 $\mathrm{mmHg}$. Her respiratory rate was 16 cycles/min, and her temperature was $37.5^{\circ} \mathrm{C}$. Physical examination revealed no meningismus but there was trismus. No wound or scar could be found on her body apart from the Lanz abdominal incision which was covered with gauze. This was opened and found to be dry. A wound swab was taken. The abdomen was rigid on palpation. Cardiopulmonary and neurological examination results were unremarkable. Pelvic examination revealed minimal dirty-white vaginal discharge. During the examination, she had a spasm. This confirmed the diagnosis of tetanus.

She was admitted into a quiet private room with the lights dimmed and prescribed intramuscular anti tetanus serum 15,000 units after a negative test dose and tetanus toxoid $0.5 \mathrm{ml}$. She was further placed on intravenous metronidazole $500 \mathrm{mg} 8$ hourly, ampicillin/cloxacillin $500 \mathrm{mg} 6$ hourly and gentamicin $80 \mathrm{mg} 8$ hourly and diazepam $10 \mathrm{mg}$ stat then $20 \mathrm{mg}$ in intravenous dextrose saline infusion 1 litre 8 hourly to sedate her. Her blood investigation and wound swab results yielded nothing of significance.

Further questioning of the relatives revealed that she had been separated from her husband after five years of marriage because of infertility. She had regularly been receiving herbal medications from a traditional birth attendant (TBA) in her locality for this problem.

Despite the level of sedation, she continued having 
occasional break through spasms and finally died, on the third day after admission, from severe hypoxia due to spasm of respiratory muscles.

\section{DISCUSSION}

Tetanus is a major public health hazard in the developing world. Despite the availability of effective preventive vaccines, it still accounts for about 1 million deaths annually, owing largely to the failure of immunization programmes in the developing countries [2-4]. In most countries it is a notifiable disease caused by Clostridium tetani which produces tetanospasmin, the neurotoxin responsible for the disease symptoms [3]. Since the days of Hippocrates, its symptomatology has remained largely unchanged, though the pathophysiology has been explored to a molecular level and options for prevention and management have changed enormously [4]. In Nigeria mortality rate of tetanus has been reported to be in the range of $26 \%$ - $60 \%$ which is similar to the global picture [2,5], and $10 \%$ of adult tetanus cases were attributed to maternal tetanus [4].

Diagnosis of tetanus involves the isolation and identification of $C$. tetani and the detection of toxigenicity in the isolate by mouse toxicity testing [6]. This is not widely acceptable as some authorities have reported that the laboratory tests generally are not helpful for diagnosing tetanus [7]. This is because the isolation of organisms from wounds is neither sensitive nor specific to tetanus [8]. Serodiagnosis is not relevant since the clinical disease does not result in the production of tetanus antitoxin. It has therefore been suggested that the diagnosis of tetanus in developing countries should be based on physical examination, medical and immunization history, and the signs and symptoms of muscle spasms, stiffness and pain [7]. The diagnosis of tetanus in this case was made based on this suggestion.

Postoperative tetanus is a calamitous complication [9], with a Nigerian prevalence of $5 \%$ in the mid 20th century [10], and $3.3 \%$ in the developed world [6]. At the turn of the 21st century, the prevalence in Nigeria has dropped to about 2\% [2], but remained at 3.5\% in the developed world [8]. Owing largely to the availability of intensive care facilities in the developed countries, the mid-20th century introduction of tetanus antitoxin and toxoid, improvement in wound care and antibiotics, the mortality rate of postoperative tetanus has declined but has remained at near $100 \%$ in Nigeria $[2,8,10]$.

Postoperative tetanus has been reported to develop quickly, usually within 24 hours [8], and this could account for the high case fatality rate (as seen in the index case), owing to the fact that the mortality is inversely related to the length of incubation period and period of onset $[5,8]$.
It is pertinent to mention that rural healthcare delivery in developing countries is fraught with material, infrastructural and human challenges. This hinders the ability of practitioners to provide the most basic surgical care to prevent morbidity and mortality. Studies in Sierra Leone [11] and other eight low-resource countries (Sri Lanka, Mongolia, Tanzania, Afghanistan, Sierra Leone, Liberia, Gambia and São Tomé and Principe) [12] found that none of the countries had $100 \%$ of its facilities reporting continuous supply of uninterrupted water, electricity, and oxygen. Confronted with these problems, the rural surgeon has to make some adaptations to be able to render services to his patients. These occasionally compromise the sterility of the operating room environment. First is the use of inappropriate ventilation in the event of failed electricity supply. This allows bacterial contaminants including dust-laden tetanus spores to enter the operating room causing infection [13]. Second is sterilization of instruments on kerosene stoves, done when there is no power supply. This is not effective enough to destroy tetanus spores which can only be destroyed by very high temperature over a long duration [14].

Patient characteristics and comorbidity play an important role in determining the likelihood of infection after surgery [15]. This can manifest as poor personal hygiene, lack of immunization and ignorance [2] which is very common in developing countries. The patient presented was a case of infertility who was ignorantly being treated by a traditional birth attendant (TBA). The activities of TBAs are known to involve the use of roots and occasionally waste products (animal dung and cow urine), flies, insertion of herbal vaginal pessaries and making of scarification marks into which some of their preparations are applied [16]. The genital tract having been reported as a portal of entry in $3.6 \%$ of cases [5] may implicate her treatment for infertility by the TBA as a possible source of infection.

\section{CONCLUSION}

Tetanus including postoperative tetanus is a largely preventable disease that still occurs globally. Considering the surgical environment in developing countries, a possible intra-operative contamination was a likely source of infection. The events in this case emphasize the advisability of active immunization of all persons against tetanus, adequate sterilization of surgical equipment and consumables as well as the operating rooms.

Ethical Issues: Necessary permission was obtained before this case was written up for publication.

\section{REFERENCES}

[1] Kasher, J.A. and Glen, M. (2007) Acquiring tetanus after haemorrhoid banding and other gastrointestinal proce- 
dures. Journal of Gastrointestinal Surgery, 11, 515-519. doi:10.1007/s11605-006-0079-6

[2] Onwuchekwa, A.C. and Asekomeh, E.G. (2009) A 10year review of outcome of management of tetanus in adults at a Nigerian tertiary hospital. Annals of African Medicine, 8, 168-172.

[3] Bartlett, J.G. (1992) Clostridium tetani. In: Infectious Diseases, W. B. Saunders, Philadelphia, 1580-1583.

[4] Attygalle, D. and Karalliedde, L. (1997) Unforgettable tetanus. European Journal of Anaesthesiology, 14, 122133. doi:10.1097/00003643-199703000-00003

[5] Patel, J.C. and Metha, B.C. (1999) Tetanus: Study of 8697 cases. Indian Journal of Medical Sciences, 53, 393401.

[6] Public Health Laboratory Network, Tetanus Laboratory Case Definition (LCD).

http://www.health.gov.au/internet/main/publishing.nsf/Co ntent/cda-phlncd-tetanus.htm

[7] Mayo Clinic (2013) Tetanus: Test and diagnosis. http://www.mayoclinic.com/health/tetanus/DS00227/DSE CTION=tests-and-diagnosis

[8] Dhalla, S. (2004) Postsurgical tetanus. Cannadian Journal of Surgery, 47, 375-379

[9] Segel, J. and Shaff, B.L. (1969) An unusual case of postoperative tetanus. South African Medical Journal, 43, 1556-1557.

[10] Johnstone, D.D. (1958) Tetanus in Nigeria: Review of 100 cases treated in Ibadan. British Medical Journal, 1, 12-16. doi:10.1136/bmj.1.5061.12
[11] Kingham, T.P., Kamara, T.B., Cherian, M.N., Gosselin, R.A., Simkins, M., Meissner, C., et al., Quantifying surgical capacity in Sierra Leone: A guide for improving surgical care. Archives of Surgery, 144, 122-127. doi:10.1001/archsurg.2008.540

[12] Kushner, A.L., Cherian, M.N., Noel, L., Spiegel, D.A., Groth, S. and Etienne, C. (2010) Addressing the millennium development goals from a surgical perspective: Essential surgery and anesthesia in 8 low- and middle-income countries. Archives of Surgery, 145, 154-159. doi:10.1001/archsurg.2009.263

[13] Mateos, I., Valencia, R., Torres, M.J., Cantos, A., Conde, M. and Aznar, J. (2006) Nosocomial outbreak of Pseudomonas aeruginosa endophthalmitis. Infection Control and Hospital Epidemiology, 27, 1249-1251. doi:10.1086/508838

[14] Pflug, I.J., Holcomb, R.G. and Gomez, M.M. (2001) Principles of the thermal destruction of microorganisms. In: Block, S.S., Ed., Disinfection, Sterilization, and Preservation. 5th Edition, Lipincott Williams and Wilkins, Philadelphia, 79-129.

[15] Dindo, D., Muller, M.K., Weber, M. and Clavien, P.A. (2003) Obesity in general elective surgery. Lancet, 361, 2032-2035. doi:10.1016/S0140-6736(03)13640-9

[16] Ofili, A.N. and Okojie, O.H. (2005) Assessment of the role of traditional birth attendants in maternal health care in Oredo Local Government Area, Edo State, Nigeria. Journal of Community Medicine and Primary Health Care, 17, 55-60. doi:10.4314/jcmphc.v17i1.32428 